\title{
FRR: a Proportional and Worst-Case Fair Round Robin Scheduler
}

\author{
Xin Yuan and Zhenhai Duan \\ Department of Computer Science \\ Florida State University \\ Tallahassee, FL 32306 \\ Email: \{xyuan,duan\}@cs.fsu.edu
}

\begin{abstract}
In this paper, we propose an $O(1)$ complexity round robin scheduler, called Fair Round Robin $(F R R)$, that provides good fairness and delay properties. Unlike existing $O(1)$ complexity round robin schedulers that can only achieve long term fairness, $F R R$ not only provides proportional fairness, but also maintains a constant normalized worst-case fair index as defined in Bennett and Zhang's work. This means that $F R R$ guarantees both short term and long term fairness among all backlogged flows.
\end{abstract}

Index Terms-System design, Deterministic network calculus

\section{INTRODUCTION}

Packet scheduling has been extensively studied in the last decade due to its importance in the provision of Quality of Service (QoS) guarantees in data networks. An ideal packet scheduler should have the following three properties. First, to be used in high speed networks, the scheduler should have a low complexity, preferably $O(1)$. Second, the scheduler should incur a bounded delay for each packet that reaches the head of the queue for a flow in order for the scheduler to support QoS guarantees. Last, the scheduler must provide fairness among the flows competing for the shared link so that each flow can get its fair share of the bandwidth.

While complexity and delay are well defined, the fairness of a scheduler needs further elaboration. Detailed discussion on this subject can be found in [8]. In this paper, we will use two well established fairness criteria, the worst-case fairness that was defined by Bennett and Zhang in [2] and the proportional fairness that was defined by Golestani in [5]. A scheduler, $s$, is worst-case fair to flow $f_{i}$ if and only if the delay of a packet arriving at $t$ on flow $f_{i}$ is bounded by $\frac{Q_{i, s}(t)}{r_{i}}+C_{i, s}$, where $Q_{i, s}(t)$ is the queue size of $f_{i}$ at $t, r_{i}$ is the guaranteed rate of $f_{i}$, and $C_{i, s}$ is a constant independent of the queues of other flows. A scheduler is worst-case fair if it is worst-case fair to all flows in the system. If a scheduler, $s$, is worst-case fair, the fairness of the scheduler is measured by the normalized worst-case fair index [2]. Let $R$ be the total link bandwidth. The normalized worst-case fair index for the scheduler, $c_{s}$, is defined as $c_{s}=\max _{i}\left\{\frac{r_{i} C_{i, s}}{R}\right\}$.

Let $S_{i, s}\left(t_{1}, t_{2}\right)$ be the amount of data of flow $f_{i}$ sent during time period $\left[t_{1}, t_{2}\right)$. For any two flows $f_{i}$ and $f_{j}$ that are backlogged during any time period $\left[t_{1}, t_{2}\right)$, the proportional

This work is partially supported by NSF grants ANI-0106706, CCR0208892, and CCF-0342540. fairness requires the difference between the normalized service received by the two flows, $\left|\frac{S_{i, s}\left(t_{1}, t_{2}\right)}{r_{i}}-\frac{S_{j, s}\left(t_{1}, t_{2}\right)}{r_{j}}\right|$, to be bounded by a small constant.

Essentially, worst-case fairness requires the guaranteed rates of all flows in the system to be enforced at all time within a small error margin, while proportional fairness requires bandwidths allocated to any two backlogged flows to be proportional to their weights (guaranteed rates) at all time within a small error margin. To achieve both long term and short term fairness, a packet scheduler should provide both worst-case fairness and proportional fairness.

Existing scheduling algorithms can be classified into two types, timestamp based approaches [1], [2], [4], [5], [10] and round-robin algorithms [6], [7], [11], [15]. Timestamp based approaches have good bounded delay and fairness properties with a relatively high complexity, $O(\log N)$, where $N$ is the number of flows in the system. The implementation of timestamp based algorithms in high speed networks is problematic due to the logarithmic complexity. Round-robin algorithms have an $O(1)$ complexity, but in general do not have good bounded delay and fairness properties. For example, none of the existing round-robin schemes can achieve a constant normalized worst-case fair index.

In this paper, we propose a new packet scheduling scheme, called Fair Round-Robin (FRR). FRR combines the ideas in timestamp based approaches and round-robin algorithms. It maintains an $\mathrm{O}(1)$ per packet scheduling complexity in that the complexity is independent of the number of flows in the system. $F R R$ has the desired properties of an ideal packet scheduler: an $\mathrm{O}(1)$ per packet processing complexity, a strict rate-proportional delay bound; and proportional and worst-case fairness for flows that are continuously backlogged.

The rest of the paper is structured as follows. Section II presents related work. Section III introduces the background of this work. Section IV describes $F R R$. Section V discusses the QoS properties of $F R R$. Section VI reports the results of the simulation study of $F R R$. In the study, $F R R$ is compared with other representative packet scheduling algorithms including Weighted Fair Queueing [10], Smoothed Round Robin [6], and Stratified Round Robin [11]. Finally, Section VII concludes the paper. 


\section{RELATED WORK}

Packet scheduling has been studied extensively and many scheduling algorithms have been proposed. Existing schedulers either use a timestamp based approach [1], [2], [4], [5], [10], [16], [19] or are round-robin algorithms [3], [6], [7], [11], [15].

Some timestamp based schedulers, such as Weighted Fair Queuing $(W F Q)$ [10] and Worst-case Fair Weighted Fair Queuing $\left(W F^{2} Q\right)$ [1], [2], closely approximate the Generalized Processor Sharing $(G P S)$ [4], [10], which is an unrealistic algorithm that achieves perfect fairness and isolation among all flows. These schedulers compute a timestamp for each packet by emulating the progress of a reference GPS server and transmit packets in the increasing order of their timestamps. Both $W F Q$ and $W F^{2} Q$ have an $O(1) G P S$-relative delay [17], that is, for each packet $p, F_{W F Q}^{p}-F_{G P S}^{p} \leq c_{1} * \frac{L_{M}}{R}$ and $F_{W F^{2} Q}^{p}-F_{G P S}^{p} \leq c_{2} * \frac{L_{M}}{R}$, where $F_{s}^{p}$ is the time when packet $p$ finishes service under scheduler $s, L_{M}$ is the maximum packet size, $R$ is the link bandwidth, and $c_{1}$ and $c_{2}$ are two constants. It has been shown in [17] that the lower bound computational complexity of any scheduling algorithm that guarantees an $O(1) G P S$-relative delay bound is $\Omega(\log N)$, where $N$ is the number of flows in the system. Fortunately, having an $O(1)$ GPS-relative delay is not necessary for a scheduler to achieve worst-case and proportional fairness and, as will be shown in this paper, designing an $O(1)$ complexity scheduler that is worst-case and proportional fair is possible. Note that having an $O(1) G P S$-relative delay is not sufficient for a scheduler to have a constant normalized worst-case fair index. For example, as shown in [2], $W F Q$ does not have a constant normalized worst-case fair index.

Other timestamp based approaches, such as Self-Clocked Fair Queuing (SCFQ) [5] and Virtual Clock [19], compute timestamps without referring to a reference GPS server. These methods still need to sort packets according to their timestamps and still have an $O(\log N)$ per packet processing complexity. The Leap Forward Virtual Clock [16] reduces the sorting complexity by coarsening timestamp values and has an $O(\log \log N)$ complexity. This scheme requires complex data structures and is not suitable for hardware implementation.

Round-robin schedulers serve backlogged flows in some kind of round-robin fashion and have an $O(1)$ per packet processing complexity. To achieve fairness with different packet sizes, the Deficit Round Robin ( $D R R)$ scheme [15] augments the traditional round robin algorithm with a deficit counter and a quantum for each flow. A number of methods have recently been proposed to improve delay and burstiness properties of $D R R$ [6], [7], [11]. The Smoothed Round Robin (SRR) scheme [6] improves the delay and burstiness properties by spreading the data of a flow to be transmitted in a round over the entire round using a weight spread sequence. Aliquem [7] allows the quantum of a flow to be scaled down, which results in better delay and burstiness properties. Both SRR and Aliquem improve the average packet delay over $D R R$, however, the worst-case single packet delay bound is proportional

\begin{tabular}{|l|l|}
\hline$N$ & the number of flows in the system \\
\hline$n$ & the number of classes in the system \\
\hline$R$ & total link bandwidth \\
\hline$r_{i}$ & guaranteed bandwidth for flow $f_{i}$ \\
\hline$w_{i}=\frac{r_{i}}{R}$ & the weight associated with flow $f_{i}$ \\
\hline$l_{M}$ & maximum packet size \\
\hline$S_{i, s}\left(t_{1}, t_{2}\right)$ & $\begin{array}{l}\text { the amount of work received by session } i \\
\text { during }\left[t_{1}, t_{2}\right) \text { under the } s \text { server }\end{array}$ \\
\hline$S_{i, s}(t)$ & $\begin{array}{l}\text { the amount of work received by session } i \\
\text { during }[0, t) \text { under the } s \text { server }\end{array}$ \\
\hline$F_{i, s}^{k}$ & $\begin{array}{l}\text { the departure time of the } k \text { th packet of } \\
\text { flow } f_{i} \text { under the } s \text { server }\end{array}$ \\
\hline$F_{s}^{p}$ & $\begin{array}{l}\text { the departure time of packet } p \text { under } \\
\text { the } s \text { server }\end{array}$ \\
\hline$Q_{i, s}(t)$ & $\begin{array}{l}\text { the queue size of flow } f_{i} \text { at time } t \text { under } \\
\text { the } s \text { server }\end{array}$ \\
\hline$p_{i}^{k}$ & the $k$ th packet on flow $f_{i}$ \\
\hline
\end{tabular}

TABLE I

NOTATION USED IN THIS PAPER

to the number of flows in the system. Like SRR, the Stratified Round Robin [11] scheme also tries to spread the data of a flow by using a deadline based scheduling scheme to ensure that all flows get their fair share of slots. Stratified Round Robin enjoys a single packet delay bound that is related to the guaranteed rate of the flow and is independent of the number of flows in the system. Bin Sort Fair Queuing (BSFQ) [3] uses an approximate bin sort mechanism to schedule packets. Each packet is assigned a timestamp (deadline). Packets are roughly sorted by placing them into bins according to their timestamps. No sorting of packets is performed within each bin. The worstcase single packet delay of BSFQ is proportional to the number of flows. Hybrid scheduling schemes [12], [13] have also been proposed, where the scheduling tasks are separated into two levels. While the algorithm components of these schemes are similar to those of $F R R$, the QoS properties of these schemes are not clear.

None of existing round-robin schemes has a constant normalized worst-cast fair index. To the best of our knowledge, our proposed scheme, $F R R$, is the first $O(1)$ time scheduler that has a constant normalized worst-cast fair index.

\section{PRELIMINARIES}

Major notations used in this paper are summarized in Table I. There are $N$ flows $f_{1}, f_{2}, \ldots, f_{N}$ sharing a link of bandwidth $R$. Each flow $f_{i}$ has a minimum guaranteed rate of $r_{i}$. We will assume that $\sum_{i=1}^{N} r_{i} \leq R$. The weight $w_{i}$ of flow $f_{i}$ is defined as its guaranteed rate normalized with respect to the total rate of the link, i.e.,

$$
w_{i}=\frac{r_{i}}{R}
$$

Thus, we have $\sum_{i=1}^{N} w_{i} \leq 1$. A scheduler determines the order of the packets to be served (transmitted), and thus, the bandwidth allocation for each flow. For example, using $G P S$, the bandwidth allocated to flow $f_{i}$ is $\frac{w_{i}}{\sum_{f_{j} \text { is backlogged }} w_{j}} R \geq r_{i}$. 


\section{A. Deficit Round Robin}

Since the proposed scheduler, $F R R$, is built over Deficit Round Robin $(D R R)$ [15], we will briefly describe $D R R$ and present some properties of $D R R$ that are needed to understand the properties of $F R R$.

Like the ordinary round robin scheme, $D R R$ works in rounds. Within each round, each backlogged flow has an opportunity to send packets. Each flow $f_{i}$ is associated with a quantity quantum $_{i}$ and a variable deficitcounter $r_{i}$. The quantity quantum $m_{i}$ is assigned based on the guaranteed rate for $f_{i}$ and specifies the target amount of data that $f_{i}$ should send in each round. However, since the scheduler operates in a packet-by-packet fashion, $f_{i}$ may not be able to send exactly quantum $_{i}$ bytes in a round. The variable deficitcounter $i$ is introduced to record the quantum that is not used in a round so that the unused quantum can be passed to the next round. To ensure that each flow can send at least one packet in a round, in this paper, we will assume that quantum $_{i}$ is larger than the maximum packet size, that is,

$$
\text { quantum }_{i} \geq L_{M} .
$$

More details about $D R R$ can be found in [15]. Some properties of $D R R$ are summarized in the following lemmas. Due to the page limit constraint, we omit the proofs of these lemmas and some other lemmas and theorems in the later of this paper. The proofs of all lemmas and theorems are available online in the technical report version of this paper [18].

Lemma 1: Assuming that flow $f_{i}$ is continuously backlogged during $\left[t_{1}, t_{2}\right)$. Let $X$ be the smallest number of continuous $D R R$ rounds that completely enclose $\left[t_{1}, t_{2}\right)$. The service received by $f_{i}$ during this period, $S_{i, D R R}\left(t_{1}, t_{2}\right)$, is given by

$$
(X-3) \text { quantum }_{i} \leq S_{i, D R R}\left(t_{1}, t_{2}\right) \leq(X+1) \text { quantum }_{i} .
$$

Proof: See [18].

Lemma 2: Let $f_{1}, \ldots, f_{N}$ be the $N$ flows in the system with guaranteed rates $r_{1}, \ldots, r_{N} \cdot \sum_{i=1}^{N} r_{i} \leq R$. Let $r_{\min }=$ $\min _{i}\left\{r_{i}\right\}$ and $r_{\text {max }}=\max _{i}\left\{r_{i}\right\}$. Assume that there exists a constant $C$ such that $r_{\max } \leq C * r_{\min }$, and that $D R R$ is used to schedule the flows with quantum $m_{i}=L_{M} * \frac{r_{i}}{r_{m i n}}$. The following statements are true.

1) Let packet $p$ arrive at the head of the queue for $f_{i}$ at time $t$. There exists a constant $c_{1}$ such that packet $p$ will be serviced before $t+c_{1} \times \frac{L_{M}}{r_{i}}$.

2) The normalized worst-case fair index of $D R R$ in such a system is a constant.

3) Let $f_{i}$ and $f_{j}$ be continuously backlogged during any given time period $\left[t_{1}, t_{2}\right)$, there exists two constants $c_{1}$ and $c_{2}$ such that the normalized service received by the two flows during this period is bounded by

$$
\left|\frac{S_{i, D R R}\left(t_{1}, t_{2}\right)}{r_{i}}-\frac{S_{j, D R R}\left(t_{1}, t_{2}\right)}{r_{j}}\right| \leq c_{1} \frac{L_{M}}{r_{i}}+c_{2} \frac{L_{M}}{r_{j}}
$$

Proof: See [18].

Lemma 2 shows that when the weights of the flows in the system are similar (within a constant factor), $D R R$ has the following three properties. First, the worst-case single packet delay depends on the guaranteed rate for the flow and is independent of the number of flows in the system. Second, $D R R$ has a constant normalized worst-case fair index, which means that $D R R$ can guarantee short term worst-case bandwidth to all flows. Third, $D R R$ provides proportional fairness. Thus, $D R R$ is an excellent scheduler when the weights of flows are similar. The problem with $D R R$ is that when the weights of the flows differ significantly, which is common in practice, the three properties do not hold simultaneously. In particular, with $D R R$, flows with large weights can be significantly affected by many flows with small weights both in terms of packet delay and short term bandwidth allocation.

Fair Round Robin $(F R R)$, our proposed scheduler, extends $D R R$ such that the three quality of service properties hold for any weight distribution, while maintaining an $O(1)$ complexity. The basic idea is as follows. $F R R$ groups flows with similar weights into classes and uses a variation of $D R R$ to schedule packets within each class. As shown in Lemma 2, $D R R$ can achieve good QoS properties for flows in each class. Thus, the challenge is to isolate the classes so that flows in different classes, which are flows with significantly different weights, do not affect each other too much. FRR uses a timestamp based scheduler to isolate the classes. As a result, $F R R$ schedules packets in two levels, a timestamp based interclass scheduling and a $D R R$ based intra-class scheduling. Since multiple flows are grouped in each class, the weight assigned to a class may change dynamically to reflect the number of active flows in the class. Traditional timestamp based approaches either cannot be directly applied or do not support provable QoS properties when weights of flows can change dynamically. A new timestamp based scheduler is developed to closely approximate $G P S$ with dynamically changing weights.

\section{FRR: A FAIR ROUND ROBIN SCHEDULER}

In this section, we will describe $F R R$, a proportional and worst-case fair round robin scheduler. Like the stratified round robin scheme [11], FRR groups flows into a number of classes with each class containing flows with similar weights. For $k \geq 1$, class $F_{k}$ is defined as

$$
F_{k}=\left\{f_{i}: \frac{1}{C^{k}} \leq w_{i}<\frac{1}{C^{k-1}}\right\}
$$

where $C$ is a constant. Let $r$ be the smallest unit of bandwidth that can be allocated to a flow. The number of classes is $n=\left\lceil\log _{C}\left(\frac{R}{r}\right)\right\rceil$. Consider an example where $R=1 T b p s$, $r=1 \mathrm{kbps}, C=8 . n=\left\lceil\log _{8}\left(10^{9}\right)\right\rceil=10$. Thus, only 10 classes are needed for this case. When $C=2, n=$ $\left\lceil\log _{2}\left(10^{9}\right)\right\rceil=30$. These examples show that the number of classes to be maintained is small in practical cases. In the rest of this paper, we will assume that the number of classes, $n$, is a small constant. Notice that $n$ is related only to $R, r$, and $C$ and is independent of $N$. Thus, even in theory, $n$ is an $O(1)$ constant with respect to $N$, the number of flows in system.

$F R R$ has two scheduling components, an intra-class scheduling that determines the order of the packets within each 
class and the weight of the class, and an inter-class scheduling that determines the class, and thus, the packet within the class, to be transmitted over the link. Next, we will describe these two components.

\section{A. Inter-class scheduling}

As discussed earlier, the inter-class scheduler, which is designed to isolate classes, is a timestamp based scheduler. Since multiple flows are grouped into each class, the rate allocated to a class may need to be changed at different times so that each flow within a class can have its fair share of bandwidth. Thus, the inter-class scheduler should be able to handle the dynamically changing weights and achieve fair sharing of bandwidth in the presence of the dynamically changing weights. Note that while GPS achieves fair sharing of bandwidth when the weights of classes change dynamically, none of the existing timestamp based schemes, which closely approximate GPS when the weights of flows do not change, can closely approximate $G P S$ when the weights change dynamically. We develop a new scheme called Dynamic Weight Worst-case Fair weighted Fair Queuing $\left(D W^{2} F^{2} Q\right)$. $D W^{2} F^{2} Q$ has the same scheduling result as $W F^{2} Q$ [2] when the weights do not change. Theorems presented later in this section show that, when the number of classes is a small constant, $D W^{2} F^{2} Q$ closely approximates $G P S$ with dynamic weights. Next, we will first discuss how to keep track of $G P S$ progress when the weights change dynamically and then describe how packets are scheduled by $D W^{2} F^{2} Q$.

Let us denote an event in the system the following: (1) the arrival of a packet to the $G P S$ server, (2) the departure of a packet from the GPS server, and (3) the weight change of a class. Let $t_{j}$ be the time at which the $j$ th event occurs. Let the time of the first arrival of a busy period be denoted as $t_{1}=0$. For each $j=2,3, \ldots$, the set of classes that are busy in the interval $\left[t_{j-1}, t_{j}\right)$ is denoted as $B_{j-1}$. Let us denote $w_{i, j-1}$ the weight for class $i$ during the interval $\left[t_{j-1}, t_{j}\right)$, which is a fixed value. The GPS progress of class $i$ during the time interval $\left[t_{j-1}, t_{j-1}+\tau\right)$, where $0<\tau \leq t_{j}-t_{j-1}$, is

$$
\begin{gathered}
S_{i, G P S}\left(t_{1}\right)=0 \\
S_{i, G P S}\left(t_{j-1}+\tau\right)=S_{i, G P S}\left(t_{j-1}\right)+\frac{w_{i, j-1}}{\sum_{k \in B_{j-1}} w_{k, j-1}} \times R \times \tau
\end{gathered}
$$

$D W^{2} F^{2} Q$ keeps track of the GPS progress of all the classes using the above formula. Notice that for each event, the GPS progress of all classes may need to be updated. The per event computational complexity is $O(n)$, where $n$ is the number of classes, which is a small constant in FRR. Thus, assuming that weight change is less frequent than packet arrival, which is true in $F R R$, the per packet computational complexity for maintaining $G P S$ progress is $O(n)=O(1)$. Since $D W^{2} F^{2} Q$ schedules packets only at packet boundaries (packet arrivals and departures), it is sufficient to maintain accurate $G P S$ progress at packet boundaries.

In addition to keeping track of $G P S$ progress, $D W^{2} F^{2} Q$ also records the amount of data of each class that have been serviced. Assume that the server needs to decide the next packet at time $t_{j}$. Let $s i z e_{i}\left(t_{j}\right)$ be the size of the packet at the head of class $i$ at time $t_{j}, S_{i, G P S}\left(t_{j}\right)$ be the amount of data of session $i$ served under $G P S$, and $S_{i, D W^{2} F^{2} Q}\left(t_{j}\right)$ be the amount of data session $i$ actually served under $D W^{2} F^{2} Q$. The head of class $i$ is scheduled at $t_{j}$ if and only if the following two conditions are met:

- Condition 1): $S_{i, G P S}\left(t_{j}\right) \geq S_{i, D W^{2} F^{2} Q}\left(t_{j}\right)$.

- Condition 2): Let $n f(i)$ be the estimated GPS finishing time of the head packet of class $i, p_{i}^{k}$. For a backlogged class $i, n f(i)$ is computed as follows. If size $_{i}\left(t_{j}\right)+$ $S_{i, D W^{2} F^{2} Q}\left(t_{j}\right)>S_{i, G P S}\left(t_{j}\right)$,

$$
\begin{gathered}
n f(i)=t_{j}+\frac{\operatorname{size}_{i}\left(t_{j}\right)+S_{i, D W^{2} F^{2} Q}\left(t_{j}\right)-S_{i, G P S}\left(t_{j}\right)}{R \times w_{i, j}} . \\
\text { If } \operatorname{size}_{i}\left(t_{j}\right)+S_{i, D W^{2} F^{2} Q}\left(t_{j}\right) \leq S_{i, G P S}\left(t_{j}\right), \\
n f(i)=F_{i, G P S}^{k} .
\end{gathered}
$$

Class $i$ has the smallest estimated GPS finishing time, $n f(i)$, among all backlogged classes. For classes with the same $n f(i)$, the class number can be used to break the tie.

The first condition enforces that $D W^{2} F^{2} Q$ does not schedule a packet before the $G P S$ starting time of the packet. This ensures that $D W^{2} F^{2} Q$ can be at most one packet ahead of $G P S$. The second condition enforces that packets are ordered based on the estimated GPS finishing time. The class whose head packet has the smallest estimated $G P S$ finishing time is scheduled. In estimating the GPS finishing time, there are two cases. The first case is when the packet has not departed under $G P S\left(\operatorname{size}_{i}\left(t_{j}\right)+S_{i, D W^{2} F^{2} Q}\left(t_{j}\right)>S_{i, G P S}\left(t_{j}\right)\right)$. In this case, the finishing time is estimated using the current weight, $w_{i, j}$. $R \times w_{i, j}$ is the GPS guaranteed rate for weight $w_{i, j}$. Note that since the weights of classes can change dynamically, this estimated GPS finishing time of a packet may be inaccurate. The second case is when the packet has departed under $G P S$ size $\left._{i}\left(t_{j}\right)+S_{i, D W^{2} F^{2} Q}\left(t_{j}\right) \leq S_{i, G P S}\left(t_{j}\right)\right)$. In this case, the actual GPS departure time, which is the accurate GPS finishing time, is used as the timestamp. Hence, $D W^{2} F^{2} Q$ uses accurate information to schedule packets that fall behind $G P S$ and may use inaccurate information to schedule packets that are ahead of $G P S$. The complexity to schedule a packet is $O(n)$, where $n$ is the number of classes, which is a small constant in FRR. Thus, the per-packet computational complexity of the inter-class scheduling is $O(n)=O(1)$. The following sequence of theorems shows some properties of $D W^{2} F^{2} Q$.

Theorem 1: $D W^{2} F^{2} Q$ is work conserving.

Proof: Since GPS is work-conserving, we will prove the theorem by showing that $D W^{2} F^{2} Q$ has the same idle and busy periods as $G P S$. Assuming that $D W^{2} F^{2} Q$ and $G P S$ have different idle and busy periods. Let $t$ be the first occurrence when $G P S$ and $D W^{2} F^{2} Q$ are not in the same state. There are two cases.

Case 1: GPS is idle and $D W^{2} F^{2} Q$ is busy, serving packet $p$. Since $t$ is the first occurrence when GPS and $D W^{2} F^{2} Q$ are not in the same state, the amount of data served during $[0, t)$ must be the same for the two scheduling schemes. Since 
$p$ is currently being served under $D W^{2} F^{2} Q$, from condition 1), $p$ must be started before $t$ under $G P S$. Since $G P S$ is idle at time $t$, packet $p$ must finish before $t$ under GPS. Hence, there must exist a packet $q$ such that $q$ has not been served under $G P S$ during $[0, t)$ and has been served by $D W^{2} F^{2} Q$ during $[0, t)$. Since $G P S$ is idle at $t$, packet $q$ should start after $t$ under $G P S$, which indicates that $q$ cannot be served under $D W^{2} F^{2} Q$ during $[0, t)$ since condition 1$)$ does not hold. This is the contradiction.

Case 2: GPS is busy and $D W^{2} F^{2} Q$ is idle. Let packets $p_{1}, p_{2}, \ldots, p_{i}$ be the packets departed under GPS during $[0, t)$ and packets $c p_{1}, \ldots, c p_{j}$ be the packets currently in progress under $G P S$. Since GPS is busy, at least one packet is being serviced at time $t$. Since $D W^{2} F^{2} Q$ is idle at $t$, all packets satisfy condition 1) should have been served, that is, packets $p_{1}, p_{2}, \ldots, p_{i}$ and $c p_{1}, \ldots, c p_{j}$ are all served during $[0, t)$ under $D W^{2} F^{2} Q$. Thus, during $[0, t), D W^{2} F^{2} Q$ sends more data than GPS and $t$ cannot be the first occurrence that GPS and $D W^{2} F^{2} Q$ are not in the same state.

Since both $G P S$ and $D W^{2} F^{2} Q$ are work-conserving disciplines, their busy periods coincide. In the rest of the section, we will consider packet scheduling within one busy period. Let $F_{i, s}^{k}$ be the departure time of the $k$ th packet in class $i$ under server $s$.

Lemma 3: If $F_{i, G P S}^{k} \leq F_{j, G P S}^{m}, F_{i, D W^{2} F^{2} Q}^{k}<F_{j, D W^{2} F^{2} Q}^{m+1}$. Proof: Let $p_{i}^{l}$ be the packet at the head of class $i$ at time $t$ when $p_{j}^{m+1}$ is at the head of class $j$ and is eligible to be transmitted. From Condition 1), $t \geq F_{j, G P S}^{m}$ and the estimate finishing time for packet $p_{j, D W^{2} F^{2} Q}^{m+1}, n f(j)>F_{j, G P S}^{m}$.

If $l>k$, we have $F_{i, D W^{2} F^{2} Q}^{k}<F_{j, D W^{2} F^{2} Q}^{m+1}$ and the lemma is proved. If $l \leq k$,

$$
F_{i, G P S}^{l}<F_{i, G P S}^{l+1}<\ldots<F_{i, G P S}^{k} \leq F_{j, G P S}^{m}<n f(j) .
$$

Since packets $p_{i}^{l}, p_{i}^{l+1}, \ldots, p_{i}^{k}$ have departed before time $t$ under $G P S$, the actual GPS departing times of the packets will be assigned as the estimated $G P S$ finishing times of these packets. Thus, all these packets will have a smaller estimated $G P S$ finishing time than packet $p_{j}^{m+1}$. Since $D W^{2} F^{2} Q$ selects the class with the smallest estimated GPS finishing time for transmission, all these packets will be transmitted before $p_{j}^{m+1}$. Thus, $F_{i, D W^{2} F^{2} Q}^{k}<F_{j, D W^{2} F^{2} Q}^{m+1}$

By allowing classes to change weights dynamically, $D W^{2} F^{2} Q$ may not be able to estimate the GPS departure time accurately since it cannot predict the future weight changes. However, Lemma 3 indicates that $D W^{2} F^{2} Q$ can at most introduce one packet difference between any two classes in comparison to GPS. This leads to the following theory that states relation of $G P S$ departure time and $D W^{2} F^{2} Q$ departure time. Let $F_{s}^{p}$ be the time packet $p$ departs under server $s$.

Theorem 2: Let $n$ be the number of classes in the system,

$$
F_{D W^{2} F^{2} Q}^{p}-F_{G P S}^{p} \leq(n-1) \frac{L_{M}}{R}
$$

Proof: Consider any busy period and let the time that it begins be time zero. Let $p_{k}$ be the $k$ th packet of size $s_{k}$ in the busy period to depart under GPS. We have

$$
F_{G P S}^{p_{k}} \geq \frac{s_{1}+s_{2}+\ldots+s_{k}}{R}
$$

Now consider the departure time of $p_{k}$ under $D W^{2} F^{2} Q$. From Lemma 3, each class can have at most one packet whose $G P S$ finishing time is after packet $p_{k}$ and whose $D W^{2} F^{2} Q$ finishing time is before packet $p_{k}$. Hence, there are at most $n-1$ packets (from the $n-1$ other classes) that depart before packet $p_{k}$ under $D W^{2} F^{2} Q$ and have a GPS finishing time after $F_{G P S}^{p_{k}}$. Let the $n-1$ packets be $e_{1}, e_{2}, \ldots, e_{n-1}$ with sizes $s e_{1}, s e_{2}, \ldots, s e_{n-1}$. All other packets depart before $p^{k}$ under $D W^{2} F^{2} Q$ must have $G P S$ finishing times earlier than $F_{G P S}^{p_{k}}$. We have

$$
F_{D W^{2} F^{2} Q}^{p_{k}} \leq \frac{s_{1}+\ldots+s_{k}+s e_{1}+\ldots+s e_{n-1}}{R}
$$

Thus,

$$
F_{D W^{2} F^{2} Q}^{p_{k}}-F_{G P S}^{p_{k}} \leq(n-1) \frac{L_{M}}{R}
$$

Theorem 2 gives the relative delay bound between GPS and $D W^{2} F^{2} Q$. The bound is proportional to the number of classes. Although this bound is in general not a good bound, when the number of classes in the system is a constant, as in $F R R$, this bound is sufficient: the packet departure times under $G P S$ and $D W^{2} F^{2} Q$ differ by at most a constant number of packets.

Theorem 3: For all time $\tau$ and class $i$,

$$
S_{i, G P S}(0, \tau)-S_{i, D W^{2} F^{2} Q}(0, \tau) \leq(n-1) * L_{M} .
$$

Proof: See [18].

Theorem 4: For all time $\tau$ and class $i$,

$$
S_{i, D W^{2} F^{2} Q}(0, \tau)-S_{i, G P S}(0, \tau) \leq L_{M} .
$$

Proof: Straightforward from Condition 1) of the $D W^{2} F^{2} Q$ scheduling scheme.

These theorems establish that $D W^{2} F^{2} Q$ closely approximates GPS for dynamic weights when the number of classes is a constant: the difference between the total service given to a particular class for the two scheduling schemes is within a constant number of packets. Notice that one key point that makes $D W^{2} F^{2} Q$ work in $F R R$ is that the number of classes in $F R R$ is a small constant.

\section{B. Intra-class scheduling}

Since the inter-classes scheduling scheme, $D W^{2} F^{2} Q$, schedule packets based on the weights of the classes, and since many flows are grouped into each class, intra-class scheduling needs to decide (1) the weights used to send packets in a class, and (2) the order of packets coming from different flows. Note that intra-class scheduling does not decide the actual time a packet is serviced. This function is performed by inter-class scheduling.

As discussed earlier, $D R R$ offers good $Q \circ S$ properties when it is used to schedule flows with similar weights. However, naively applying $D R R$ in the intra-class scheduling 


\begin{tabular}{|l|l|}
\hline variable & explanation \\
\hline deficitcount \\
\hline remaindeficit & the deficit count for flow $f_{i}$ \\
\hline lastingflowlist & the $D R R$ round \\
the flows that last to the next frame \\
\hline framesize & the size of the frame \\
\hline frameweight & the weight for the frame \\
\hline remainsize & $\begin{array}{l}\text { size of the part of a packet that } \\
\text { belongs to current frame }\end{array}$ \\
\hline
\end{tabular}

TABLE II

MAJOR VARIABLES USED IN THE FRAME CALCULATION ALGORITHM

does not yield a fair scheduler even assuming that the interclass scheduler is GPS. In order to obtain a fair scheduler, the intra-class scheduler must be able to transfer the fairness at the class level (provided by $D W^{2} F^{2} Q$ ) to the fairness at the flow level. In $F R R$, intra-class scheduling uses a frame based approach. The packet stream within a class is partitioned into logical frames with packets in each frame being scheduled using the same weight. The intra-class scheduling scheme is called Lookahead Deficit Round Robin with Weight Adjustment ( $L D R R W A$ ), a variation of $D R R$. In $L D R R W A$, backlogged flows are served in a round-robin fashion. To offset the weight differences among the flows in a class, each flow $f_{i} \in F_{k}=\left\{f_{i}: \frac{1}{C^{k}} \leq w_{i}<\frac{1}{C^{k-1}}\right\}$ is assigned a quantum of

$$
\text { quantum }_{i}=C^{k} w_{i} L_{M} \text {. }
$$

Since $\frac{1}{C^{k}} \leq w_{i}<\frac{1}{C^{k-1}}$

$$
L_{M} \leq \text { quantum }_{i}<C \times L_{M} .
$$

A $L D R R W A$ frame is related to, but different from, a $D R R$ round. A $L D R R W A$ frame is basically a $D R R$ round plus some packets that are in the next $D R R$ round and are moved into the current frame by the lookahead operation. Each $L D R R W A$ frame, together with its associated weight, is computed using the algorithm shown in Fig. 1. The major variables used in the algorithm are summarized in Table II. Like $D R R$, variable deficitcount ${ }_{i}$ is associated with flow $f_{i}$ to maintain the credits to be passed over to the next $D R R$ round and decide the amount of data to be sent in one round. After each $D R R$ round, remaindeficit maintains the sum of the quanta not used in the current $D R R$ round, that is, the quanta that cannot be used since the size of the next backlogged packet is larger than the remaining quanta for a flow. In traditional $D R R$, these unused quanta will be passed to the next $D R R$ round. In $L D R R W A$, in addition to passing the unused quanta to the next $D R R$ round, some packets that would be sent in the next $D R R$ round are placed in the current $L D R R W A$ frame so that at frame boundaries remainde ficit is always equal to 0 . This is the lookahead operation. As will be shown later, this lookahead operation is the key to ensure that each frame is properly sized such that long lasting flows can get their fair share of the bandwidth. The lastingflowlist contains the list of flows that are backlogged at the end of the current $D R R$ round. Flows in lasting flowlist are candidates

\section{Algorithm for computing the next frame for class $F_{k}$}

(1) remaindeficit $=$ framesize $=0$

(2) lasting flowlist $=N U L L$

(3) if (remainsize $>0$ ) then /*The partial packet belongs to this frame */

(4) framesize $=$ framesize + remainsize

\section{(5) end if}

/* forming the $D R R$ round */

(6) for each active flow $f_{i}$ do

(7) deficitcount $_{i}=$ deficitcount $_{i}+$ quantum $_{i}$

(8) while (de ficitcount $\left._{i}>0\right)$ and $\left(f_{i}\right.$ not empty) do

(9) pktsize $=\operatorname{size}\left(\right.$ head $\left.\left(f_{i}\right)\right)$

(10) if $\left(\right.$ pktsize $<$ deficitcount $\left._{i}\right)$ then

(11) remove head from $f_{i}$ and put it in the frame

(12) $\quad$ framesize $=$ framesize + pktsize

(13) de ficitcount $_{i}=$ deficitcount $_{i}-$ pktsize

(14) else break

(15) end if

(16) end while

(17) if $\left(f_{i}\right.$ is empty ) then

(18) deficitcount $_{i}=0$

(19) else

(20) $\quad$ remaindeficit $=$ remaindeficit + de $_{\text {ficitcount }}$

(21) insert $f_{i}$ to lasting flowlist

(22) end if

(23) end for

/* lookahead operation */

(24) $f_{i}=$ head(lastingflowlist)

(25) while $\left(f_{i} \neq N U L L\right)$ and (remaindeficit $\left.>0\right)$ do

(26) pktsize $=$ size $\left(\right.$ head $\left.\left(f_{i}\right)\right)$

(27) if (pktsize < remaindeficit) then

(28) remove head from $f_{i}$ and put it in the frame

(29) $\quad$ framesize $=$ framesize + pktsize

(30) remaindeficit $=$ remaindeficit - pktsize

(31) deficitcount $_{i}=$ deficitcount $_{i}-$ pktsize

(32) else break

(33) end if

(34) $f_{i}=$ nextflow $\left(f_{i}\right)$

(35) end while

(36) if $\left(f_{i} \neq N U L L\right)$ then

(37) pktsize $=$ size $\left(\right.$ head $\left.\left(f_{i}\right)\right)$

(38) remove head from $f_{i}$ and put it in the frame

(39) framesize $=$ framesize + remaindeficit

(40) remainsize = pktsize - remaindeficit

(41) deficitcount $_{i}=$ deficitcount $_{i}-$ pktsize

(42) end if

/* computing the weight $* /$

(43) weight $=$ totalquantum $=0$

(44) for each active flow $f_{i}$ in current frame do

(45) weight $=$ weight $+w_{i}$

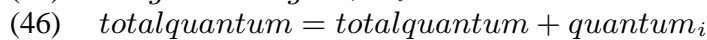

(47) end for

(48) frameweight $=$ weight $* \frac{\text { framesize }}{\text { totalquantum }}$

(49) if $\left(\right.$ frameweight $\left.<\frac{1}{C^{k}}\right)$ frameweight $=\frac{1}{C^{k}}$

Fig. 1. The algorithm for computing the next frame for class $F_{k}$ 
to supply packets for the lookahead operation. Frameweight is the weight to be used by inter-class scheduling for the current frame. Variable framesize records the size of the current frame. FRR needs this information to determine when to invoke the frame computation algorithm to compute the next frame (and thus, when to change weights for classes). Note that frame boundaries may not align with packet boundaries since $F R R$ needs to enforce that remainde ficit $=0$ at frame boundaries. Thus, a packet may belong to two frames (in the simulated GPS, weight change may happen within a packet). Variable remainsize is the size of the part of the last packet in the frame that belongs to the next frame, and thus, should be counted in the framesize for the next frame. In FRR, frame is a logical concept that affects only the progress of the simulated GPS. Thus, not aligning frame boundaries with packet boundaries does not cause problems in the actual packet scheduling.

Let us now examine the algorithm in Fig. 1. In the initialization phase, line (1) to line (5), variables are initialized and remainsize is added to framesize, which effectively includes the partial packet in the frame to be computed. After the initialization, there are three main components in the algorithm: forming a $D R R$ round, lookahead operation, and weight calculation. In the first component, line (6) to line (23), the algorithm puts all packets in the current $D R R$ round that have not been served into the current frame. In the second component, line (24) to line (42), the algorithm performs the lookahead operation by moving some packets in the next $D R R$ round into the current frame so that remaindeficit $=$ 0 at the frame boundary. This is done by allowing some flows to borrow credits from the next $D R R$ round. Since remaindeficit $=0$, no credit is passed from one frame to the next frame for the class that aggregates many flows. Notice that each backlogged flow can contribute at most one packet in the lookahead operation. Notice also that a class as a whole does not pass credits between frames. However, for an individual flow, credits may still pass from one frame to the next. As a result, the deficitcount f $_{i}$ variable may have a negative or positive value at frame boundaries.

The last component in the algorithm, line (43) to line (49) calculates the weight for the frame. As will be proved in the following lemmas, the weights are assigned such that (1) the weight for the frame is always less than or equal to the sum of all weights of the active flows in the frame (Lemma 5) and (2) the service time for each frame for class $F_{k}$ is at most $\frac{C^{k} L_{M}}{R}$ (Lemma 6). Notice that $\frac{C^{k} L_{M}}{R}=\frac{C^{k} * w_{i} * L_{M}}{w_{i} * R}=\frac{\text { quantum }}{r_{i}}$. $\frac{C^{k} L_{M}}{R}$ is the "standard" time for a flow in $F_{k}$ to send its quantum using its guaranteed rate.

The complexity of the algorithm is $O(M)$, where $M$ is the number of packets in a frame. Hence, the amortised per packet complexity for frame construction is $O(1)$. This algorithm is invoked in two occasions: (1) when the class becomes backlogged (when a packet arrives at an idle class), and (2) when the current frame is finished under the simulated GPS. To invoke the algorithm at the time when the current frame is finished under the simulated $G P S$, a timer is associated with each class. The timers record the estimated GPS finishing times of the current frames. Every time a frame departs or arrives under $G P S$, the timers for all classes are updated to reflect the changing of the GPS progress and the changing of weights. When a timer expires, the corresponding frame is finished under GPS and the algorithm is invoked to compute a new frame. Maintaining the timers results in $O(n)=O(1)$ per frame overhead. Next, we will prove a sequence of lemmas that show the properties of $L D D R W A$.

Lemma 4: Assuming that flow $f_{i}$ is continuously backlogged during $\left[t_{1}, t_{2}\right)$. Let $X$ be the smallest number of continuous $L D R R W A$ frames that completely enclose $\left[t_{1}, t_{2}\right)$. The service received by $f_{i}$ during this period, denoted as $S_{i, L D R R W A}\left(t_{1}, t_{2}\right)$, is given by

$(X-4)$ quantum $_{i} \leq S_{i, L D R R W A}\left(t_{1}, t_{2}\right) \leq(X+2)$ quantum $_{i}$.

Proof: The notation $S_{i, L D R R W A}\left(t_{1}, t_{2}\right)$ is abused in this lemma since $L D R R W A$ does not decide the actual timing to service packets. In this lemma, $S_{i, L D R R W A}\left(t_{1}, t_{2}\right)$ denotes the amount of data for a continuously backlogged flow $f_{i}$ in $X$ continuous $L D R R W A$ frames (of a particular class) using any inter-class scheduling scheme.

Since $f_{i}$ is continuously backlogged, it will try to send as many packets as possible in each frame. Since $X$ frames enclose $\left[t_{1}, t_{2}\right)$, flow $f_{i}$ will fully utilize at least $X-2$ frames (all but the first frame and the last frame). In the $X-2$ frames, $(X-2) \times$ quantum $_{i}$ credits are generated for flow $f_{i}$. The lookahead operation in the frame prior to the $X-2$ frames may borrow at most one packet, whose size is less than $L_{M}$, from $f_{i}$ in the first of the $X-2$ frames and flow $f_{i}$ in the last of the $X-2$ frames may pass at most $L_{M}$ credits to the next frame. Note that the lookahead operation borrows at most one packet from each backlogged flow. Note also that, while frames for a class do not pass credits between each other accumulatively for all flows in the class, an individual flow may pass credits across frame boundaries. Thus,

$$
S_{i, L D R R W A}\left(t_{1}, t_{2}\right) \geq(X-2) \times \text { quantum }_{i}-L_{M}-L_{M} .
$$

Since quantum $i \geq L_{M}$,

$$
S_{i, L D R R W A}\left(t_{1}, t_{2}\right) \geq(X-4) \times \text { quantum }_{i} .
$$

On the other hand, $f_{i}$ will be serviced in at most all the $X$ frames, which produces $X \times$ quantum $_{i}$ credits for $f_{i}$ during this period of time. Flow $f_{i}$ in the frame prior to the $X$ frames may have at most $L_{M}$ left-over credits and the lookahead operation in the last of the $X$ frames may borrow at most $L_{M}$ credits from $f_{i}$ in the next frame. Thus,

$$
\begin{aligned}
S_{i, L D R R W A}\left(t_{1}, t_{2}\right) & \leq X \times \text { quantum }_{i}+L_{M}+L_{M} \\
& \leq(X+2) \text { quantum }_{i} .
\end{aligned}
$$

Comparing Lemma 4 and Lemma 1, we can see the similarity between $D R R$ and $L D R R W A$. However, as we will show in the following lemmas, unlike $D R R$ rounds, $L D R R W A$ 
frames are always properly sized so that continuously backlogged flows can obtain their fair shares of the bandwidth.

Lemma 5: The weight for a frame is less than or equal to the sum of the weights of the active flows in the frame.

Proof: Since no credit is passed between frames, the amount of data that is allowed to send in one frame is at most the total quanta of all active flows generated in that frame. Thus, framesize $\leq \sum_{f_{i}}$ quantum $_{i}$. From line (48) in Figure 1, frameweight $=\sum_{f_{i}} w_{i} * \frac{\text { framesize }}{\sum_{f_{i}} \text { quantum } m_{i}} \leq \sum_{f_{i}} w_{i}$. If the condition in line (49) is true, frameweight is assigned to the minimum weight of a flow in the class and thus the same conclusion holds.

For any given time, let $c w_{i}, 1 \leq i \leq n$ be the weights for the $n$ classes $\left(c w_{i}\right.$ may change over time). Lemma 5 establishes that $\sum_{i=1}^{n} c w_{i} \leq \sum_{i=1}^{N} w_{i} \leq 1$. Thus, under GPS (with dynamic weights), the bandwidth allocated to class $i$ is given by

$$
\frac{c w_{i}}{\sum_{i=1}^{n} c w_{i}} R \geq R \times c w_{i} .
$$

We will call $R \times c w_{i}$ the GPS guaranteed rate since this rate is guaranteed when the weight for a class is $c w_{i}$ regardless how other classes change weights.

Lemma 6: Under $G P S$, the time to service each $L D R R W A$ frame in class $F_{k}$ is at most $C^{k} \frac{L_{M}}{R}$.

Proof: Consider first that the condition in line (49) is not true and the frame weight is computed in line (48) in Fig. 1. In this case,

$$
\begin{aligned}
& \text { frameweight }=\sum_{f_{i}} w_{i} * \frac{\text { framesize }}{\sum_{f_{i}} \text { quantum }_{i}} \\
& =\sum_{f_{i}} w_{i} * \frac{f_{f_{i}} \text { ramesize }}{\sum_{f_{i}} C^{k} w_{i} L_{M}}=\frac{\text { framesize }}{C^{k} L_{M}}
\end{aligned}
$$

Thus, the GPS guaranteed rate for this class is $R \frac{\text { framesize }}{C^{k} L_{M}}$ and the total time to serve this frame is at most

$$
\frac{\text { framesize }}{R \frac{\text { framesize }}{C^{k} L_{M}}}=\frac{C^{k} L_{M}}{R} .
$$

If the condition in line (49) is true, the weight for the frame is increased and the conclusion still holds.

Lemma 7: Under GPS, the time to service $X$ bytes of data in the queue for class $F_{k}$ is at most $\frac{X C^{k}}{R}$.

Proof: The minimum weight assigned to a backlogged class $F_{k}$ is $\frac{1}{C^{k}}$. Thus, the GPS guaranteed rate for class $F_{k}$ is at least $\frac{R}{C^{k}}$. Thus, the time to serve a queue of size $\mathrm{X}$ bytes in class $F_{k}$ is at most $\frac{X}{\frac{R}{C^{k}}}=\frac{X C^{k}}{R}$.

Lemma 8: For a class $F_{k}$ frame of size no smaller than $L_{M}$, the service time for the frame is exactly $C^{k} \frac{L_{M}}{R}$ using the $G P S$ guaranteed rate.

Proof: When the frame is larger than $L_{M}$, frameweight $=$ $\frac{\text { framesize }}{C^{k} L_{M}} \geq \frac{1}{C^{k}}$. Thus, the GPS guaranteed rate for the frame is $R \frac{\text { framesize }}{C^{k} L_{M}}$ and the service time for the frame with the guaranteed rate is

$$
\frac{\text { framesize }}{R \frac{\text { framesize }}{C^{k} L_{M}}}=\frac{C^{k} L_{M}}{R} .
$$

Lemma 9: Let a class $F_{k}$ frame contains packets of a continuously backlogged flow $f_{i}$, the size of frame is no smaller than $L_{M}$.

Proof: Straight-forward from the fact that no credit is passed from the previous frame and to the next frame and that quantum $_{i} \geq L_{M}$.

Lemma 10: Let $f_{i} \in F_{k}$ and $f_{j} \in F_{m}$ be continuously backlogged during $\left[t_{1}, t_{2}\right) . k \geq m$. Let $X_{k}$ and $X_{m}$ be the smallest numbers of $F_{k}$ and $F_{m}$ frames that completely enclose $\left[t_{1}, t_{2}\right)$. Assume that classes $F_{k}$ and $F_{m}$ are serviced with the GPS guaranteed rate.

$$
\left(X_{k}-1\right) C^{k-m} \leq X_{m} \leq X_{k} C^{k-m}+1 .
$$

Proof: Since $f_{i} \in F_{k}$ and $f_{j} \in F_{m}$ are continuously backlogged during $\left[t_{1}, t_{2}\right)$, the sizes of all frames during this period are no smaller than $L_{M}$ (Lemma 9). From Lemma 8, using the $G P S$ guaranteed rate, the time to service a class $F_{k}$ frame is exactly $\frac{C^{k} L_{M}}{R}$ and the time for a class $F_{m}$ frame is exactly $\frac{C^{m} L_{M}}{R}$. Since $X_{k}$ and $X_{m}$ are the smallest numbers of $F_{k}$ and $\stackrel{R}{F}_{m}$ frames that completely enclose $\left[t_{1}, t_{2}\right)$, we have

$$
\begin{aligned}
t 2-t 1 & \leq X_{k} \frac{C^{k} L_{M}}{R} \leq t 2-t 1+\frac{C^{k} L_{M}}{R} \\
t 2-t 1 & \leq X_{m} \frac{C^{m} L_{M}}{R} \leq t 2-t 1+\frac{C^{m} L_{M}}{R} .
\end{aligned}
$$

Hence, $\left(X_{k}-1\right) C^{k-m} \leq X_{m} \leq X_{k} C^{k-m}+1$.

Lemma 11 relaxes the condition in Lemma 10 by not requiring each class to be serviced with its GPS guaranteed rate.

Lemma 11: Let $f_{i} \in F_{k}$ and $f_{j} \in F_{m}$ be continuously backlogged during $\left[t_{1}, t_{2}\right) . k \geq m$. Let $X_{k}$ and $X_{m}$ be the smallest numbers of $F_{k}$ and $F_{m}$ frames that completely enclose $\left[t_{1}, t_{2}\right)$. Assume that the inter-class scheduler is GPS.

$$
\left(X_{k}-1\right) C^{k-m} \leq X_{m} \leq X_{k} C^{k-m}+1 \text {. }
$$

Proof: This lemma relaxes the condition in Lemma 10 by not requiring each class to be serviced with its GPS guaranteed rate. Since $f_{i} \in F_{k}$ and $f_{j} \in F_{m}$ be continuously backlogged during $\left[t_{1}, t_{2}\right)$, the sizes of all frames during this period are no smaller than $L_{M}$ (Lemma 9). Let us partition the duration $\left[t_{1}, t_{2}\right)$ into smaller intervals $\left[a_{1}=t_{1}, b_{1}\right),\left[a_{2}=b_{1}, b_{2}\right), \ldots$, $\left[a_{Y}=b_{Y-1}, b_{Y}=t_{2}\right)$ such that within each interval $\left[a_{h}, b_{h}\right)$, $1 \leq h \leq Y$, the weights of all classes are fixed. Let $F_{1}, \ldots, F_{n}$ be the $n$ classes in the system. Let class $F_{k}$ have weight $w_{k}^{h}$ during interval $\left[a_{h}, b_{h}\right), 1 \leq h \leq Y$ (If $F_{k}$ is not backlogged, $\left.w_{k}^{h}=0\right)$. The amount of class $F_{k}$ data sent during $\left[a_{h}, b_{h}\right)$ is thus,

$$
\frac{w_{k}^{h}}{\sum_{i=1}^{n} w_{i}^{h}} R *\left(b_{h}-a_{h}\right) .
$$

Consider a reference scheduling system that contains three classes $R F_{k}, R F_{m}$, and $R F_{o}$. Let us use intervals $\left[a a_{1}=\right.$ $\left.t_{1}, b b_{1}\right),\left[a a_{2}=b b_{1}, b b_{2}\right), \ldots,\left[a a_{Y}=b b_{Y-1}, b b_{Y}\right)$ to emulate the behavior of classes $F_{k}$ and $F_{m}$ during intervals $\left[a_{1}=\right.$ $\left.t_{1}, b_{1}\right),\left[a_{2}=b_{1}, b_{2}\right), \ldots,\left[a_{Y}=b_{Y-1}, b_{Y}\right)$ respectively. Let $r w_{k}^{h}$ be the weight for class $R F_{k}$ during interval $\left[a a_{h}, b b_{h}\right)$, 
$1 \leq h \leq Y$. Let $r w_{m}^{h}$ be the weight for class $R F_{m}$ during interval $\left[a a_{h}, b b_{h}\right), 1 \leq h \leq Y$. Let $r w_{o}^{h}$ be the weight for class $R F_{o}$ during interval $\left[a a_{h}, b b_{h}\right), 1 \leq h \leq Y$. The weights and the duration of each interval are given as follows:

$$
r w_{k}^{h}=w_{k}^{h}, r w_{m}^{h}=w_{m}^{h}, r w_{o}^{h}=1-w_{k}^{h}-w_{m}^{h}, 1 \leq h \leq Y
$$

and

$$
b b_{h}=a a_{h}+\frac{b_{h}-a_{h}}{\sum_{i=1}^{n} w_{i}^{h}}, 1 \leq h \leq Y .
$$

It can be verified that the amount of classes $R F_{k}$ and $R F_{m}$ data sent in an interval $\left[a a_{h}, b b_{h}\right), 1 \leq h \leq Y$, is exactly the same as the amount of classes $F_{k}$ and $F_{m}$ data sent in an interval $\left[a_{h}, b_{h}\right), 1 \leq h \leq Y$, respectively. In an interval $\left[a a_{h}, b b_{h}\right), 1 \leq h \leq Y$, let us further assume that Class $R F_{k}$ has exactly the same sequence of packets as Class $F_{k}$ has in interval $\left[a_{h}, b_{h}\right)$ and that Class $R F_{m}$ has exactly the same sequence of packets as Class $F_{m}$ has in interval $\left[a_{h}, b_{h}\right)$. The progress of classes $F_{k}$ and $F_{m}$ during $\left[t_{1}, t_{2}\right)$ is exactly the same as the progress of class $R F_{k}$ and $R F_{m}$ during $\left[a a_{1}, b b_{Y}\right)$

In the reference system, classes $R F_{m}$ and $R F_{k}$ are serviced with the $G P S$ guaranteed rate during $\left[a a_{1}, b b_{Y}\right)$. Let $R X_{k}$ and $R X_{m}$ be the smallest numbers of $R F_{k}$ and $R F_{m}$ frames that completely enclose $\left[a a_{1}, b b_{Y}\right)$. From Lemma 10,

$$
\left(R X_{k}-1\right) C^{k-m} \leq R X_{m} \leq R X_{k} C^{k-m}+1 .
$$

Let $X_{k}$ and $X_{m}$ be the smallest number of $F_{k}$ and $F_{m}$ frames that completely enclose $\left[t_{1}, t_{2}\right)$. Since the progress of classes $F_{k}$ and $F_{m}$ during $\left[t_{1}, t_{2}\right)$ is exactly the same as the progress of class $R F_{k}$ and $R F_{m}$ during $\left[a a_{1}, b b_{Y}\right)$, we have $X_{k}=R X_{k}$ and $X_{m}=R X_{m}$. Thus,

$$
\left(X_{k}-1\right) C^{k-m} \leq X_{m} \leq X_{k} C^{k-m}+1 .
$$
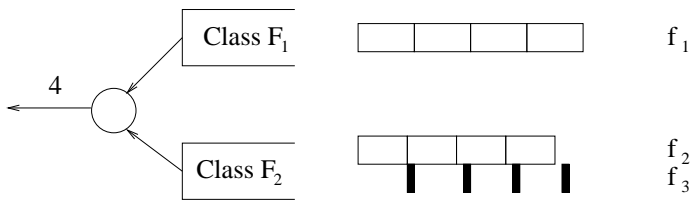

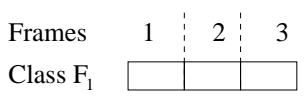

weight $=1 / 2$

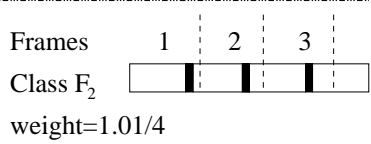

Fig. 2. An example
In the following, we will use an example to illustrate how $L D R R W A$ interacts with inter-class scheduling and how it transfers the fairness at the class level to the flow level. To simplify the discussion, we will assume that GPS is the interclass scheduling algorithm. Consider scheduling for a link with 4 units of bandwidth with the following settings. $C=2$ and there are two classes where $F_{1}=\left\{f_{i}: \frac{1}{2} \leq w_{i}<1\right\}$ and $F_{2}=\left\{f_{i}: \frac{1}{4} \leq w_{i}<\frac{1}{2}\right\}$. Three flows, $f_{1}, f_{2}$ and $f_{3}$, with rates $r_{1}=2$ and $r_{2}=r_{3}=1$ are in the system. $w_{1}=1 / 2$, $w_{2}=1 / 4$, and $w_{3}=1 / 4$. Thus, $f_{1}$ is in $F_{1}$, and $f_{2}$ and $f_{3}$ are in $F_{2}$. Let $L$ be the maximum packet size. The quantum for each of the three flows is $L$. All packets in $f_{1}$ are of size $L$, all packets in $f_{2}$ are of size $0.99 \mathrm{~L}$ and all packets in $f_{3}$ are of size $0.01 L$. Flows $f_{1}$ and $f_{2}$ are always backlogged. Flow $f_{3}$ is not always backlogged, its packets arrive in such a way that exactly one packet arrives before a new frame is to be formed. Thus, each $F_{2}$ frame contains one packet from $f_{3}$. The example is depicted in Fig. 2. As shown in the figure, each $F_{1}$ frame contains exactly one packet from $f_{1}$. For $F_{2}$, the lookahead operation always moves part of the $f_{2}$ packet in the next $D R R$ round into the current frame, and thus, the frame boundaries are not aligned with packet boundaries. Note that frames from different classes are not aligned. We will shows how worst-case and proportional fairness for flows $f_{1}$ and $f_{2}$ is achieved.

The weight for $F_{1}$, which is computed using line (48) in Fig. 1, is always $1 / 2$. For $F_{2}$, the lookahead operation ensures that the size of $f_{2}$ data in a frame is $L$, and thus, the size of each $F_{2}$ frame is $L+0.01 L=1.01 L$. The weight of $F_{2}$ is computed using line (48) in Fig. 1. weight $=\left(w_{2}+w_{3}\right) *$

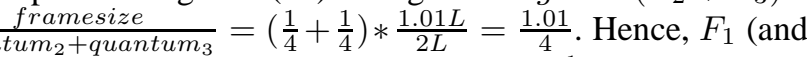
thus $f_{1}$ ) is allocated a bandwidth of $4 * \frac{\frac{1}{2}}{\frac{1}{2}+\frac{1.01}{4}}=\frac{8}{3.01}>2$. $F_{2}$ is allocated a bandwidth of $4 * \frac{\frac{1.01}{4}}{\frac{1}{2}+\frac{1.01}{4}}$. For each $f_{2}$ frame of size $1.01 L, L$ belongs to $f_{2}$. Thus, the rate allocated to $f_{2}$ is $4 * \frac{\frac{1.01}{4}}{\frac{1}{2}+\frac{1.01}{4}} * \frac{L}{1.01 L}=\frac{4}{3.01}>1$. The rates allocated to $f_{1}$ and $f_{2}$ are larger than their guaranteed rates and the worstcase fairness is honored. Furthermore, the ratio of the rates allocated to $f_{1}$ and $f_{2}$ is equal to $\frac{\frac{8}{3.01}}{\frac{4}{301}}=2$, which is equal to the ratio of their weights. Thus, the proportional fairness is also honored. In the next section, we will formally prove that $F R R$ has worst-case and proportional fairness.

\section{Properties OF FRR}

This session analyzes fairness and delay properties of $F R R$. We will prove that the three statements in Lemma 2 hold for $F R R$ with an arbitrary weight distribution.

Theorem 5 (single packet delay bound): Let packet $p$ arrives at the head of flow $f_{i} \in F_{k}$ at time $t$. Using $F R R$, there exists a constant $c_{1}$ such that $p$ will depart before $t+c_{1} * \frac{L_{M}}{r_{i}}$.

Proof: If class $F_{k}$ is idle under GPS at time $t$, a new frame that includes packet $p$ will be formed at time $t$. From Lemma 6 , under $G P S$, the frame will be serviced at most at time $t+C^{k} \frac{L_{M}}{R} \leq t+C \frac{L_{M}}{r_{i}}$. Hence, from Theorem 2, the frame will be serviced under $D W^{2} F^{2} Q$ before $t+C \frac{L_{M}}{r_{i}}+(n-1) \frac{L_{M}}{R} \leq$ $t+(C+n-1) \frac{L_{M}}{r_{i}}$, where $n$ is the number of classes in the system. Thus, there exists $c_{1}=C+n-1$ such that packet departs before $t+c_{1} * \frac{L_{M}}{r_{i}}$.

If class $F_{k}$ is busy under GPS at time $t$, packet $p$ will be included in the frame that is computed the next time the frame computation algorithm is invoked, which is at the end of the current frame under consideration by GPS. From Lemma 6, $F_{G P S}^{p} \leq t+2 * \frac{L_{M} C^{k}}{R} \leq t+\frac{2 C L_{M}}{r_{i}}$. From Theorem 2, the frame will be serviced under $D W^{2} F^{2} Q$ before $t+2 C \frac{L_{M}}{r_{i}}+(n-$ 
1) $\frac{L_{M}}{R} \leq t+(2 C+n-1) \frac{L_{M}}{r_{i}}$. Thus, there exists $c_{1}=2 C+n-1$ such that packet $p$ departs before $t+c_{1} * \frac{L_{M}}{r_{i}}$.

Theorem 5 shows that like the stratified round robin scheme [11], FRR also has a worst-case single packet delay bound that is only related to requested rate of the flow and is independent of the number of flows in the system. Next, we will consider the worst-case fairness property of $F R R$.

Theorem 6 (worst-case fairness): $F R R$ has a constant normalized worst-case fairness index.

Proof: Let a packet belonging to flow $f_{i} \in F_{k}$ arrive at time $t$, creating a total backlog of $q_{i}$ bytes in $f_{i}$ 's queue. Let packet $p_{1}$ be the first packet in the backlog. From the proof of Theorem 5, we have $F_{G P S}^{p_{1}} \leq t+2 C \frac{L_{M}}{r_{i}}$. After the first packet is serviced under $G P S$, from Lemma 4, at most $\left\lceil\frac{q_{i}}{\text { quantum }}\right\rceil+4 \leq \frac{q_{i}}{\text { quantum }}+5$ frames will be needed to drain the queue. From Lemma 6, under GPS, servicing the $\frac{q_{i}}{\text { quantum } i}+5$ frames will take at most

$$
\begin{aligned}
\left(\frac{q_{i}}{\text { quantum }}+5\right) * C^{k} \frac{L_{M}}{R} & =\frac{q_{i}}{C^{k} w_{i} L_{M}} \frac{C^{k} L_{M}}{R}+5 C^{k} \frac{L_{M}}{R} \\
& \leq \frac{q_{i}}{r_{i}}+5 C \frac{L_{M}}{r_{i}}
\end{aligned}
$$

Thus, under $G P S$, the queue will be drained before $t+$ $\frac{q_{i}}{r_{i}}+2 C \frac{L_{M}}{r_{i}}+5 C \frac{L_{M}}{r_{i}}$. From Theorem 2, under $D W^{2} F^{2} Q$, the queue will be drained before $t+\frac{q_{i}}{r_{i}}+7 C \frac{L_{M}}{r_{i}}+(n-1) \frac{L_{M}}{R}$. Thus, there exists a constant $d=7 C+n-1$ such that the queue will be drained before $t+\frac{q_{i}}{r_{i}}+d \frac{L_{M}}{r_{i}}$ and the normalized worstcase fair index for $F R R$ is $\max _{i}\left\{\frac{r_{i} * d \frac{L_{M}}{r_{i}}}{R}\right\}=d \frac{L_{M}}{R}$, which is a constant.

To the best of our knowledge, $F R R$ is the first $\mathrm{O}(1)$ complexity scheduling scheme that has a constant worst-case fairness index. Next we will consider $F R R$ 's proportional fairness.

Lemma 12: Assuming that $f_{i} \in F_{k}$ and $f_{j} \in F_{m}$ are continuously backlogged during $\left[t_{1}, t_{2}\right), k \geq m$. Assume that the inter-class scheduler is GPS and the intra-class scheduler is $L D R R W A$. Let $S_{i}\left(t_{1}, t_{2}\right)$ be the services given to flow $f_{i}$ during $\left[t_{1}, t_{2}\right)$ and $S_{j}\left(t_{1}, t_{2}\right)$ be the services given to flow $f_{j}$ during $\left[t_{1}, t_{2}\right)$. There exists two constants $c_{1}$ and $c_{2}$ such that

$$
\left|\frac{S_{i}\left(t_{1}, t_{2}\right)}{r_{i}}-\frac{S_{j}\left(t_{1}, t_{2}\right)}{r_{j}}\right| \leq \frac{c_{1} * L_{M}}{r_{i}}+\frac{c_{2} * L_{M}}{r_{j}} .
$$

Proof: Let $X_{k}$ and $X_{m}$ be the smallest numbers of $F_{k}$ and $F_{m}$ frames that completely enclose $\left[t_{1}, t_{2}\right)$. Since $f_{i}$ and $f_{j}$ are continuously backlogged during the $\left[t_{1}, t_{2}\right)$ period, from Lemma 4 , the services given to $f_{i}$ and $f_{j}$ during this period satisfy:

$$
\left(X_{k}-4\right) \text { quantum }_{i} \leq S_{i}\left(t_{1}, t_{2}\right) \leq\left(X_{k}+2\right) \text { quantum }_{i}
$$

and

$$
\left(X_{m}-4\right) \text { quantum }_{j} \leq S_{j}\left(t_{1}, t_{2}\right) \leq\left(X_{m}+2\right) \text { quantum }_{j} .
$$

The conclusion follows by manipulating these in-equations and applying Lemma 11, which gives the relation between $X_{k}$ and $X_{m}$,

$$
\left(X_{k}-1\right) C^{k-m} \leq X_{m} \leq X_{k} C^{k-m}+1 .
$$

In the following, we will derive the bound for $\frac{S_{i}\left(t_{1}, t_{2}\right)}{r_{i}}-$ $\frac{S_{j}\left(t_{1}, t_{2}\right)}{r_{j}}$. We will have the term $C O N$ to represent the general term $c_{1} \frac{L_{M}}{r_{i}}+c_{2} \frac{L_{M}}{r_{j}}$, where $c_{1}$ and $c_{2}$ are constants.

$$
\begin{aligned}
& \frac{S_{i}\left(t_{1}, t_{2}\right)}{r_{i}}-\frac{S_{j}\left(t_{1}, t_{2}\right)}{r_{j}} \\
\leq & \frac{\left(X_{k}+2\right) \text { quantum }_{i}}{r_{i}}-\frac{\left(X_{m}-4\right) \text { quantum }_{j}}{r_{j}} \\
\leq & \frac{\text { quantum }_{i} X_{k}}{r_{i}}-\frac{\text { quantum }_{j} X_{m}}{r_{j}}+C O N \\
\leq & \frac{\text { quantum }_{i} X_{k}}{r_{i}}-\frac{\text { quantum }_{j}\left(X_{k}-1\right) C^{k-m}}{r_{j}}+C O N \\
= & \frac{\text { quantum }_{i} X_{k}}{r_{i}}-\frac{\text { quantum }_{j}\left(X_{k}\right) C^{k-m}}{r_{j}} \\
& \quad+\frac{\text { quantum }_{j} C^{k-m}}{r_{j}}+C O N
\end{aligned}
$$

We have $\frac{q u a n t u m_{j} C^{k-m}}{r_{j}}=\frac{C^{m} w_{j} L_{M} C^{k-m}}{w_{j} R} \leq \frac{C * L_{M}}{r_{i}}$ and quantum $_{i} X_{k}-\underline{\text { quantum }_{j}\left(X_{k}\right) C^{k-m}}$

$$
=\frac{C^{k} w_{i} L_{M} X_{k}}{w_{i} R}-\frac{C^{m} w_{j} L_{M} X_{k} C^{k-m}}{w_{j} R}=0
$$

Thus, $\frac{S_{i}\left(t_{1}, t_{2}\right)}{r_{i}}-\frac{S_{j}\left(t_{1}, t_{2}\right)}{r_{j}} \leq C O N$. The bound for $\frac{S_{j}\left(t_{1}, t_{2}\right)}{r_{j}}$ $-\frac{S_{i}\left(t_{1}, t_{2}\right)}{r_{i}}$ can be derived in a similar fashion.

Lemma 12 shows that if $G P S$ is used as the interclass scheduling algorithm, the scheduling algorithm provides proportional fairness. Since $D W^{2} F^{2} Q$ approximates $G P S$, we will show in the next theorem that $F R R$, which uses $D W^{2} F^{2} Q$ as the inter-class scheduling algorithm, also supports proportional fairness.

Theorem 7 (proportional fairness): In any time period $\left[t_{1}, t_{2}\right)$ during which flows $f_{i} \in F_{k}$ and $f_{j} \in F_{m}$ are continuously backlogged in $F R R$. There exists two constants $c_{1}$ and $c_{2}$ such that

$$
\left|\frac{S_{i, F R R}\left(t_{1}, t_{2}\right)}{r_{i}}-\frac{S_{j, F R R}\left(t_{1}, t_{2}\right)}{r_{j}}\right| \leq \frac{c_{1} * L_{M}}{r_{i}}+\frac{c_{2} * L_{M}}{r_{j}} .
$$

Proof: There are two cases. The first case is when flows $f_{i}$ and $f_{j}$ are in the same class, that is, $k=m$. The second case is when flows $f_{i}$ and $f_{j}$ are not in the same class, that is, $k \neq m$. The proof of the first case is similar to the proof of the statement 3 in Lemma 2. Here, we will focus on the second case. Let us assume that $k>m$.

Let packets $p_{k}^{1}, p_{k}^{2}, \ldots, p_{k}^{a}$ be the sequence of class $F_{k}$ packets sent under $F R R$ during $\left[t_{1}, t_{2}\right)$. Let packets $p_{m}^{1}, p_{m}^{2}, \ldots, p_{m}^{b}$ be the sequence of class $F_{m}$ packets sent under $F R R$ during $\left[t_{1}, t_{2}\right)$. Since flows $f_{i}$ and $f_{j}$ are continuously backlogged during $\left[t_{1}, t_{2}\right)$, there exists a packet $p_{k}^{0}$ that departed before $p_{k}^{1}$ and $p_{k}^{a+1}$ that will depart after $p_{k}^{a}$. Under the simulated $G P S$, there is no idle time between packet $p_{k}^{0}$ and packet $p_{k}^{1}$ and between packet $p_{k}^{a}$ and packet $p_{k}^{a+1}$. Packets $p_{m}^{0}$ and $p_{m}^{b+1}$ are defined similarly.

Consider the progress of these packets under the simulated $G P S$. Let $B_{G P S}^{p}$ denote the beginning time of packet $p$ under GPS and $F_{G P S}^{p}$ denote the finishing time of packet $p$ under GPS. There are four cases: (1) $B_{G P S}^{p_{k}^{1}} \geq B_{G P S}^{p_{m}^{1}}$ and $F_{G P S}^{p_{k}^{a}}<F_{G P S}^{p_{m}^{b}}$, (2) $B_{G P S}^{p_{k}^{1}} \geq B_{G P S}^{p_{m}^{1}}$ and $F_{G P S}^{p_{k}^{a}} \geq F_{G P S}^{p_{m}^{b}}$, (3) $B_{G P S}^{p_{k}^{1}}<B_{G P S}^{p_{m}^{1}}$ and $F_{G P S}^{p_{k}^{a}}<F_{G P S}^{p_{m}^{b}}$, and (4) $B_{G P S}^{p_{k}^{1}}<B_{G P S}^{p_{m}^{1}}$ and $F_{G P S}^{p_{k}^{a}} \geq F_{G P S}^{p_{m}^{b}}$. 


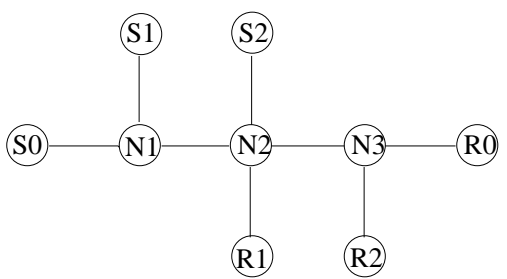

Fig. 3. Simulated network.

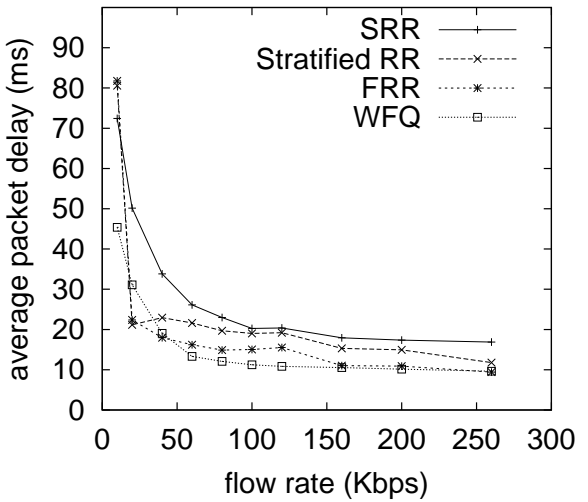

Fig. 4. Average end-to-end delay.

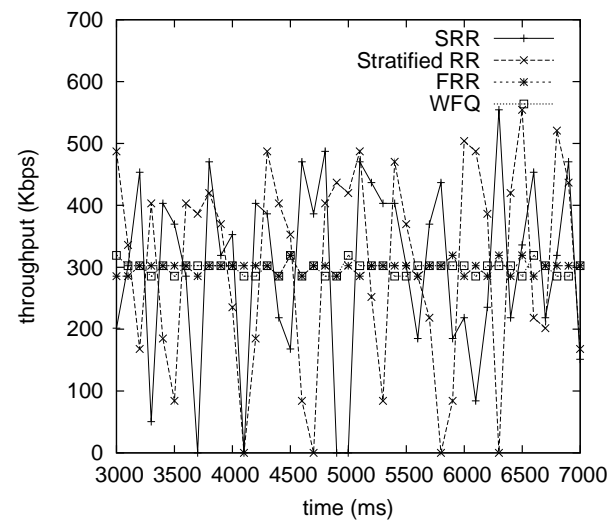

Fig. 5. Short-term throughput
In the next, we will prove case (1). Other three cases can be proven in a similar fashion. Consider case (1) when

$$
B_{G P S}^{p_{k}^{1}} \geq B_{G P S}^{p_{m}^{1}} \text { and } F_{G P S}^{p_{k}^{a}}<F_{G P S}^{p_{m}^{b}}
$$

Let $t t_{0}=B_{G P S}^{p_{m}^{1}}, t t_{1}=B_{G P S}^{p_{k}^{1}}, t t_{2}=F_{G P S}^{p_{k}^{a}}$, and $t t_{3}=$ $F_{G P S}^{p_{m}^{b}}$. We have $t t_{0} \leq t t_{1} \leq t t_{2} \leq t t_{3}$. Let $S_{i, G P S}\left(t_{1}, t_{2}\right)$ be the services that flow $f_{i}$ received during time $\left[t_{1}, t_{2}\right)$ in the simulated GPS. We have

$$
\begin{gathered}
S_{i, F R R}\left(t_{1}, t_{2}\right)=S_{i, G P S}\left(t t_{1}, t t_{2}\right) \\
S_{j, F R R}\left(t_{1}, t_{2}\right)=S_{j, G P S}\left(t t_{0}, t t_{1}\right)+S_{j, G P S}\left(t t_{1}, t t_{2}\right)+S_{j, G P S}\left(t t_{2}, t t_{3}\right)
\end{gathered}
$$

In the simulated GPS system, flows $f_{i}$ and $f_{j}$ are continuously backlogged during $\left[t t_{1}, t t_{2}\right)$. From Lemma 12, there exist two constants $c c_{1}$ and $c c_{2}$ such that

$$
\left|\frac{S_{i, G P S}\left(t t_{1}, t t_{2}\right)}{r_{i}}-\frac{S_{j, G P S}\left(t t_{1}, t t_{2}\right)}{r_{j}}\right| \leq \frac{c c_{1} * L_{M}}{r_{i}}+\frac{c c_{2} * L_{M}}{r_{j}} .
$$

Thus,

$\left|\frac{S_{i, F R R}\left(t_{1}, t_{2}\right)}{r_{i}}-\frac{S_{j, F R R}\left(t_{1}, t_{2}\right)}{r_{j}}\right|$

$\leq\left|\frac{S_{i, G P S}\left(t t_{1}, t t_{2}\right)}{r_{i}}-\frac{S_{j, G P S}\left(t t_{1}, t t_{2}\right)}{r_{j}}\right|+\frac{S_{j, G P S}\left(t t_{0}, t t_{1}\right)}{r_{j}}+\frac{S_{j, G P S}\left(t t_{2}, t t_{3}\right)}{r_{j}}$

$\leq \frac{c c_{1} * L_{M}}{r_{i}}+\frac{c c_{2} * L_{M}}{r_{j}}+\frac{S_{j, G P S}\left(t t_{2}, t t_{3}\right)}{r_{j}}+\frac{S_{j, G P S}\left(t t_{0}, t t_{1}\right)}{r_{j}}$

Next, we will consider the two terms $\frac{S_{j, G P S}\left(t t_{0}, t t_{1}\right)}{r_{j}}$ and $\frac{S_{j, G P S}\left(t t_{2}, t t_{3}\right)}{r_{j}}$. First, consider class $F_{m}$ packets serviced during $\left[t t_{0}, t t_{1}\right)$. Since all these packets are serviced after packet $p_{k}^{0}$ under $F R R\left(D W^{2} F^{2} Q\right.$ as the inter-class scheduler), from Lemma 3, at most one of the packets can have a GPS finishing time before $F_{G P S}^{p_{k}^{0}}=B_{G P S}^{p_{k}^{1}}=t t_{1}$. That is, there can be at most one class $F_{m}$ packet finishing during $\left[t t_{0}, t t_{1}\right)$. Thus, in the simulated $G P S$, at most two class $F_{m}$ packets can be serviced during $\left[t t_{0}, t t_{1}\right)$ and

$$
\frac{S_{j, G P S}\left(t t_{0}, t t_{1}\right)}{r_{j}} \leq \frac{2 L_{M}}{r_{j}} .
$$

Now, consider class $F_{m}$ packets serviced during $\left[t t_{2}, t t_{3}\right)$. Since all these packets are serviced under $F R R$ before packet $p_{k}^{a+1}$, at most one of the packets can have a $G P S$ finishing time after $F_{G P S}^{p_{k}^{a+1}}$. From Lemma 7, the duration of packet $p_{k}^{a+1}$ is less than $\frac{{ }^{m}{ }^{m} L_{M}}{R}$ in the simulated $G P S$, which is less than one frame whose size is larger than $L_{M}$. Let $X$ be the number of frames for class $F_{m}$ during this period when $p_{k}^{a+1}$ is in progress under $G P S$. Since $f_{j}$ is continuously backlogged during this period of time, from Lemma $11, X \leq C^{k-m} * 1+1$. Thus, from Lemma 4 , during the period that packet $p_{k}^{a+1}$ is in progress under $G P S$, the amount of services given to flow $f_{j}$ is at most $\left(C^{k-m}+1+2\right)$ quantum $_{j}$.

$$
\begin{aligned}
\frac{S_{j, G P S}\left(t t_{2}, t t_{3}\right)}{r_{j}} & \leq \frac{\left(C^{k-m}+1+2\right) q^{2 u a n t u m_{j}}+L_{M}}{r_{j}} \\
& =\frac{\left(C^{k-m}+3\right) w_{j} C^{m} L_{M}+L_{M}}{w_{j} R} \leq \frac{L_{M} C^{k}}{R}+\frac{3 L_{M} C^{m}}{R}+\frac{L_{M}}{r_{j}} \\
& \leq C \frac{L_{M}}{r_{i}}+(3 C+1) \frac{L_{M}}{r_{j}}
\end{aligned}
$$

Thus, there exists two constants $c_{1}=c c_{1}+C$ and $c_{2}=$ $c c_{2}+2+3 C+1$ such that

$$
\left|\frac{S_{i, F R R}\left(t_{1}, t_{2}\right)}{r_{i}}-\frac{S_{j, F R R}\left(t_{1}, t_{2}\right)}{r_{j}}\right| \leq \frac{c_{1} * L_{M}}{r_{i}}+\frac{c_{2} * L_{M}}{r_{j}} .
$$

\section{Simulation EXPERIMENTS}

In this section, we report the results of our simulation experiments. These experiments are designed to investigate $F R R$ properties in practical situations and to compare $F R R$ with other scheduling disciplines, including Weighted Fair Queueing $(W F Q)$ and two recently proposed deficit round robin $(D R R)$ based schemes, Smoothed Round Robin $(S R R)$ [6] and STratified Round Robin $(S T R R)$ [11]. All experiments are performed using $n s-2$ [9], to which we added $W F Q$, $S T R R$, and FRR queuing classes. While we carried out extensive simulations, we will only report the results of two representative experiments, one for end-to-end delay and the other one for short-term throughput. Fig. 3 shows the network topology used in the experiments. All the links have a bandwidth of $2 \mathrm{Mbps}$ and a propagation delay of $1 \mathrm{~ms}$.

The first experiment shows the end-to-end delay for flows with different rates. In this experiment, there are $10 C B R$ flows from $S 0$ to $R 0$ with average rates of $10 \mathrm{Kbps}, 20 \mathrm{Kbps}$, 40Kbps, 60Kbps, 80Kbps, 100Kbps, 120Kbps, 160Kbps, $200 K$ bps, and $260 K$ bps. The packet delay of these ten CBR flows are measured. In addition to the ten observed flows, the background traffic in the system is as follows. There are five exponential on/off flows from $S 1$ to $R 1$ with rates $60 \mathrm{Kbps}$, $80 \mathrm{Kbps}, 100 \mathrm{Kbps}, 120 \mathrm{Kbps}$, and $160 \mathrm{Kbps}$. The on-time and the off-time are 0.5 second. There are five Pareto on/off flows from $S 2$ to $R 2$ with rates $60 \mathrm{Kbps}, 80 \mathrm{Kbps}, 100 \mathrm{Kbps}$, 
$120 \mathrm{Kbps}$, and $160 \mathrm{Kbps}$. The on-time and the off-time are 0.5 second. The shape parameter of the Pareto flows is 1.5 . Two $7.8 \mathrm{Kbps}$ FTP flows with infinite traffic are also in the system, one from $S 1$ to $R 1$ and the other one from $S 2$ to $R 2$. CBR flows have a fixed packet size of 210 bytes, and all other flows have a fixed packet size uniformly chosen between 128 bytes and 1024 bytes.

Fig. 4 shows the average end-to-end delays for the ten $C B R$ flows. From the figure we can see that FRR achieves average end-to-end delays that are close to the ones that can be provided by $W F Q$, especially for flows with large rates (above $150 \mathrm{Kbps}$ in the experiment). In $F R R$, the timestamp based inter-class scheduling mechanism is added on top of $D R R$ so that flows with small rates do not significantly affect flows with large rates. Thus, in a way, $F R R$ gives preference to flows with larger weights in comparison to other $D R R$ bases schemes. In this experiment, $F R R$ results in smaller average end-to-end delays than $S R R$ and $S T R R$ when the flow rate is larger than $10 \mathrm{Kbps}$, while having a larger packet delay for the $10 \mathrm{Kbps}$ flow.

The second experiment is designed to demonstrate that $F R R$ has a better short-term throughput property than existing $D R R$ based schemes. As discussed earlier, in all existing $D R R$ based schemes, the short-term throughput of a flow with a large rate can be significantly affected by flows with small rates. In this experiment, we observe one $300 K b p s C B R$ flow and one $600 \mathrm{Kbps}$ flow from $S 0$ to $R 0$. In addition, we have $5010 \mathrm{Kbps} C B R$ flows from $S 0$ and $R 0$. The background flows are the same as the previous experiment.

Fig. 5 shows the short-term thoughput of the $300 \mathrm{Kbps}$ flow with different scheduling schemes. The results for the $600 \mathrm{Kbps}$ flow show a similar trend. Each point in the figure represents the throughput in an interval of $100 \mathrm{~ms}$. As can be seen from the figure, the short term throughputs for both $S R R$ and $S T R R$ exhibit heavy fluctuations. The flow may significantly under-perform or over-perform for a period of up to $400 \mathrm{~ms}$. For example, $S R R$ significantly under-performs between $5800 \mathrm{~ms}$ and $6200 \mathrm{~ms}$ and $S T R R$ significantly overperforms between $4700 \mathrm{~ms}$ and $5100 \mathrm{~ms}$. On the other hand, $W F Q$ and $F R R$ yield much better short term thoughputs: within each interval of $100 \mathrm{~ms}$, the throughputs are always close to the ideal rate. This experiment demonstrates that $F R R$ has a better short-term throughput property than $S R R$ and $S T R R$ and is immune to impacts of many low-speed flows on the high-speed flows.

\section{CONCLUSION}

In this paper, we have described a new scheduling algorithm, Fair Round Robin (FRR). We demonstrate that FRR has the desired properties of an ideal packet scheduler: an $\mathrm{O}(1)$ per packet processing complexity, a strict rate-proportional delay bound, and proportional and worst-case fairness. In particular, $F R R$ has much better short term fairness than other recently proposed $D R R$ based schemes including smoothed round robin and stratified round robin. The constant factor in the per packet processing complexity of $F R R$ is fairly large. We are currently investigating techniques to reduce the constant factor.

\section{REFERENCES}

[1] J. Bennett and H. Zhang, "Hierarchical Packet Fair Queueing Algorithms," in ACM SIGCOMM'96 (1996).

[2] J. Bennett and H. Zhang, " $W F^{2} Q$ : Worst Case Fair Weighted Fair Queuing", in IEEE INFOCOM'96 (1996), pages 120-128.

[3] S.Cheung and C. Pencea, "BSFQ: Bin Sort Fair Queuing," in IEEE INFOCOM'O2 (2002).

[4] A. Demers, S. Keshav, and S. Shenker, "Analysis and Simulation of a Fair Queuing Algorithm," in ACM SIGCOMM'89 (1989).

[5] S. Golestani, "A Self-clocked Fair Queueing Scheme for Broadband Applications", in IEEE INFOCOM'94, 1994.

[6] C. Guo, "SRR, an O(1) Time Complexity Packet Scheduler for Flows in Multi-Service Packet Networks," in ACM SIGCOMM'01 (2001).

[7] L Lenzini, E. Mingozzi, and G. Stea, "Aliquem: a Novel DRR Implementation to Achieve Better Latency and Fairness at O(1) Complexity," in IWQoS'02 (2002).

[8] L. Massouli and J. Roberts, "Bandwidth Sharing: Objectives and Algorithms," IEEE/ACM Trans. on Networking, Vol. 10, No. 3, pages 320328, June 2002.

[9] "The Network Simulator - ns-2," available from http://www.isi.edu/nsnam/ns.

[10] A. Parekh and R. Gallager, "A Generalized Processor Sharing Approach to Flow Control in Integrated Services Networks: the Single Node Case," IEEE/ACM Transaction on Networking, Vol. 1, No. 3, pages 344-357, June 1993.

[11] S. Ramabhadran and J. Pasquale, "Stratified Round Robin: A Low Complexity Packet Scheduler with Bandwidth Fairness and Bounded Delay," in ACM SIGCOMM'03 (2003), pages 239-249.

[12] J. Rexford and A. Greenberg and F. Bonomi, "Hardware-Efficient Fair Queueing Architectures for High-Speed Networks," IEEE INFOCOM'96, 1996.

[13] J. L. Rexford, F. Bonomi, A. Greenberg, A. Wong, "A Scalable Architecture for Fair Leaky-Bucket Shaping," in INFOCOM'97 (1997), pages 1056-1064.

[14] D. Stiliadis and A. Varma, "Design and Analysis of Frame-based Fair Queueing: A New Traffic Scheduling Algorithm for Packet-Switched Networks," in ACM SIGMETRICS'96, (1996).

[15] M. Shreedhar and G. Varghese, "Efficient Fair Queuing using Deficit Round Robin," in ACM SIGCOMM'95 (1995), pages 231-242.

[16] S. Suri, G. Varghese, and G Chandranmenon, "Leap Forward Virtual Clock: An $O(\log \log N)$ Queuing Scheme with Guaranteed Delays and Throughput Fairness," in IEEE INFOCOM'97 (1997).

[17] J. Xu and R. J. Lipton, "On Fundamental Tradeoffs between Delay Bounds and Computational Complexity in Packet Scheduling Algorithms," in ACM SIGCOMM'O2 (2002).

[18] X. Yuan and Z. Duan, "FRR: a Proportional and Worst-Case Fair Round Robin Scheduler," Technical Report TR-040201, Department of Computer Science, Florida State Univerity, Feb. 2004. http://www.cs.fsu.edu/ xyuan/frrtr.pdf

[19] L. Zhang, "Virtual Clock: A New Traffic Control Scheme for Packet Switching Networks", in ACM SIGCOMM'90 (1990). 